Supporting information

\title{
Suppressed Ion Migration in Reduced-Dimensional Perovskites
}

\section{Improves Operating Stability}

Ziru Huang, ${ }^{1}$ Andrew H. Proppe, ${ }^{1,2}$ Hairen Tan, ${ }^{1,3}$ Makhsud I. Saidaminov, ${ }^{1}$ Furui Tan,,${ }^{1,4}$ Anyi Mei, ${ }^{1,5}$ Chih-Shan Tan, ${ }^{1}$ Mingyang Wei, ${ }^{1}$ Yi Hou, ${ }^{1}$ Hongwei Han, ${ }^{5}$ Shana O. Kelley,,${ }^{2,6}$ Edward H. Sargent ${ }^{1 *}$

${ }^{1}$ Department of Electrical and Computer Engineering, University of Toronto, 35 St.George Street, Toronto, Ontario M5S 1A4, Canada.

${ }^{2}$ Department of Chemistry, University of Toronto, 80 St. George Street, Toronto, Ontario, M5S 3G4, Canada.

${ }^{3}$ National Laboratory of Solid State Microstructures, Collaborative Innovation Centre of Advanced Microstructures, Jiangsu Key Laboratory of Artificial Functional Materials, College of Engineering and Applied Sciences, Nanjing University, 210093 Nanjing, Jiangsu, China.

${ }^{4}$ Key Lab of Photovoltaic Materials, Department of Physics and Electronics, Henan University, 475004 Kaifeng, China.

${ }^{5}$ Michael Grätzel Center for Mesoscopic Solar Cells, Wuhan National Laboratory for Optoelectronics, Huazhong University of Science and Technology, Wuhan 430074, Hubei, China.

${ }^{6}$ Department of Pharmaceutical Sciences, Leslie Dan Faculty of Pharmacy, University of Toronto, Toronto, Ontario, M5S 3M2, Canada, 
*Corresponding author. E-mail: ted.sargent@utoronto.ca

\section{Materials and Methods}

\section{$\underline{\text { Chemicals }}$}

CsI powder (99.999\%) and anhydrous solvents were purchased from Sigma-Aldrich. PbI2 beads (99.999\%), $\mathrm{PbBr} 2$ powder (99.999\%) and $\mathrm{PbCl}_{2}$ powder (99.999\%) were purchased from Alfa Aesar. FAI and MAI were purchased from GreatCell Solar. All salts and solvents were used as received without further purification.

\section{$\underline{\text { Preparation of perovskite solution }}$}

The Cl-capped $\mathrm{TiO}_{2}$ was prepared following the procedure reported elsewhere. ${ }^{\mathrm{S} 1}$

For the CsMAFA perovskite, CsI, MAI, FAI, $\mathrm{PbI}_{2}, \mathrm{PbCl}_{2}$ and $\mathrm{PbBr}_{2}$ were dissolved in a DMSO/DMF (1:4) mixture solution in the following molar ratios: $\mathrm{PbI}_{2} / \mathrm{PbBr}_{2} / \mathrm{PbCl}_{2}=0.955: 0.02: 0.04, \mathrm{CsI} / \mathrm{FAI} /$ $\mathrm{MAI}=0.05: 0.905: 0.045$ and $(\mathrm{FAI}+\mathrm{MAI}+\mathrm{CsI}) /\left(\mathrm{PbI}_{2}+\mathrm{PbBr}_{2}+\mathrm{PbCl}_{2}\right)=1: 1$ to get the final concentration of 1.4 M. For 2.5D perovskite, we add ALAI for $<n>=40$ and keep (ALAI+FAI + MAI $+\mathrm{CsI}) /\left(\mathrm{PbI}_{2}+\right.$ $\left.\mathrm{PbBr}_{2}+\mathrm{PbCl}_{2}\right)=41: 40$. The concentration is $1.2 \mathrm{M}$ because of lower carrier transport ability.

Spiro solution was prepared in chlorobenzene with $65 \mathrm{mg} / \mathrm{mL}$ Spiro-OMeTAD and $20 \mu \mathrm{L} / \mathrm{mL}$ tertbutylpyridine, as well as $70 \mu \mathrm{L} / \mathrm{mL}$ bis(trifluoromethane)sulfonimide lithium salt $(170 \mathrm{mg} / \mathrm{mL}$ in acetonitrile).

\section{$\underline{\text { Perovskite solar cell fabrication }}$}

Pre-patterned indium tin oxide (ITO, TFD Devices) coated glass was cleaned by sequential sonication in acetone and isopropanol. After drying the ITO substrates, Cl-capped $\mathrm{TiO}_{2}$ nanoparticles were spin-coated 
onto ITO substrates at a spin speed of $3000 \mathrm{rpm}$ for $30 \mathrm{~s}$ (two layers total to achieve the desired thickness), and then annealed on a hot plate at $150^{\circ} \mathrm{C}$ for $30 \mathrm{~min}$ in ambient air. A detailed preparation method of the Cl-capped $\mathrm{TiO}_{2}$ solution is reported elsewhere. ${ }^{35}$ These substrates were then brought into a nitrogen-filled glovebox for all subsequent spin coating and deposition steps. The perovskite films were deposited onto the $\mathrm{TiO}_{2}$ substrates with a two-step spin coating procedure. The first step was $1000 \mathrm{rpm}$ for $10 \mathrm{~s}$ with an acceleration of $200 \mathrm{rpm} / \mathrm{s}$, and the second step was $6000 \mathrm{rpm}$ for $30 \mathrm{~s}$ with a ramp-up of $2000 \mathrm{rpm} / \mathrm{s}$. Chlorobenzene $(200 \mu \mathrm{L})$ was dropped onto the spinning substrate during the second spin-coating step in the last $10 \mathrm{~s}$ of the second spin coating step. The substrates were then immediately transferred onto a hotplate and annealed at $100^{\circ} \mathrm{C}$ for $30 \mathrm{~min}$. For the post-treatment, a solution of $10 \mathrm{mg} / \mathrm{mL}$ allylammonium iodide (ALAI) in IPA was spin coated onto annealed perovskite films, followed by additional annealing for 10 minutes. The spiro-OMeTAD layer was deposited by dropping $70 \mu \mathrm{L}$ of spiro solution (preparation described above) and spin coating at 4,000 rpm for 30 s. Finally, a $120 \mathrm{~nm}$ Au contact was deposited by electron-beam evaporation (Angstrom Engineering). Encapsulation of the perovskite film for PL measurement was done using a solution of $50 \mathrm{mg} / \mathrm{ml}$ of PMMA in chlorobenzene, spin coated at $3000 \mathrm{rpm}$.

\section{$\underline{\text { Solar cell characterization }}$}

The $J-V$ characteristics were measured using a Keithley 2400 sourcemeter under the illumination with a solar simulator (Newport, class A) at an intensity $100 \mathrm{~mW} / \mathrm{cm}^{2}$ in $\mathrm{N}_{2}$ atmosphere (Sciencetech class A). Unless otherwise stated, the $J-V$ curves were measured with a scanning rate of $100 \mathrm{mV} \mathrm{s}^{-1}$ (voltage step of $10 \mathrm{mV}$ and delay time of $100 \mathrm{~ms}$ ). An aperture shade mask with an area of $4.9 \mathrm{~mm}^{2}$ was placed in front of the solar cell device to avoid overestimation of the photocurrent density. The steady-state PCE, PCE(t), was measured by setting the bias voltage to the value at maximum power point $\left(V_{\mathrm{MPP}}\right)$ and then periodically measuring the current density. $V_{\text {MPP }}$ was determined from the forward and reverse scanned $J-V$ curves. A spectral mismatch factor of 1 was used for all $J-V$ measurements. EQE measurements were performed using Newport QUANTX-300 with monochromatic light and white bias light ( $\sim 0.1$ Sun). The photodiode used for the calibration of EQE measurements has been calibrated by Newport. Stability and ageing tests at 
continuous MPP operation under 1 Sun, AM 1.5G illumination were carried out in nitrogen by fixing the voltage at $V_{\text {MPP }}$ and then tracking the current output. A 420-nm cutoff UV-filter was applied in front of the solar cells during the MPP tracking and aging tests. The cells were purged with nitrogen flow for 1 hour before MPP tracking to get rid of residual moisture on the surface.

\section{Other characterizations}

High-resolution SEM images were obtained using the Hitachi S-5200 microscope with an accelerating voltage of $5 \mathrm{kV}$. XRD patterns were obtained using a Rigaku MiniFlex 600 diffractometer equipped with an $\mathrm{NaI}$ scintillation counter and using monochromatized Copper K $\alpha$ radiation $(\lambda=1.5406 \AA)$. Photoluminescence (PL) spectra and lifetimes were measured using a Horiba Fluorolog time correlated single-photon-counting system with photomultiplier tube detectors. The excitation source was a laser diode at a wavelength of $504 \mathrm{~nm}$. The pulse duration is $110 \mathrm{ps}$, peak power per area is $400 \mathrm{~W} / \mathrm{cm}^{2}$, the average power per area is $0.468 \mathrm{~W} / \mathrm{cm}^{2}$ for $\mathrm{PL}$ aging. TOF-SIMS was performed using ToF-SIMS5 from ION-TOF GmbH. (Munster, Germany). Samples were analyzed in dual beam profiling mode. All profiles were performed in non-interlaced mode.

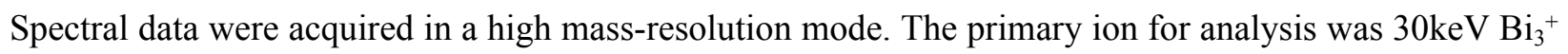
at $0.3 \mathrm{pA}$ (Bi liquid metal ion source). This ion beam was applied over a $100 \mu \mathrm{m} \times 100 \mu \mathrm{m}$ area at the center of the sputter crater. The sputter ion was $1 \mathrm{keV} \mathrm{Ar}^{+}$at $120 \mathrm{nA}$ (Ar, electron impact ion source). A 400um x 400um sputter crater was used. During the sputtering process, the mass spectra within the same layer is quite consistent and different layers have well defined profiles, which means the beam damage is not observed. X-ray Photoelectron Spectroscopy (XPS) measurements were carried out using a Thermo Scientific Al Ka source XPS system, with a $75 \mathrm{eV}$ pass energy and a spot size of $400 * 400 \mu \mathrm{m}$. Binding energy steps of $0.05 \mathrm{eV}$ were used for all measurements. Sputtering was performed with $1 \mathrm{keV} \mathrm{Ar}^{+}$ion source for one increment of 300 seconds (to dig through the bulk of the gold pixel) and then 10 subsequent steps of 30 seconds each to obtain an incremental depth profile. 
a

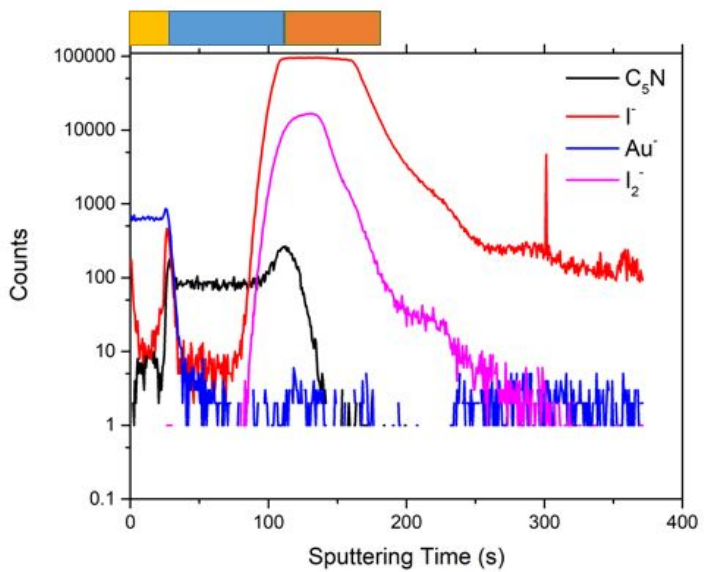

b

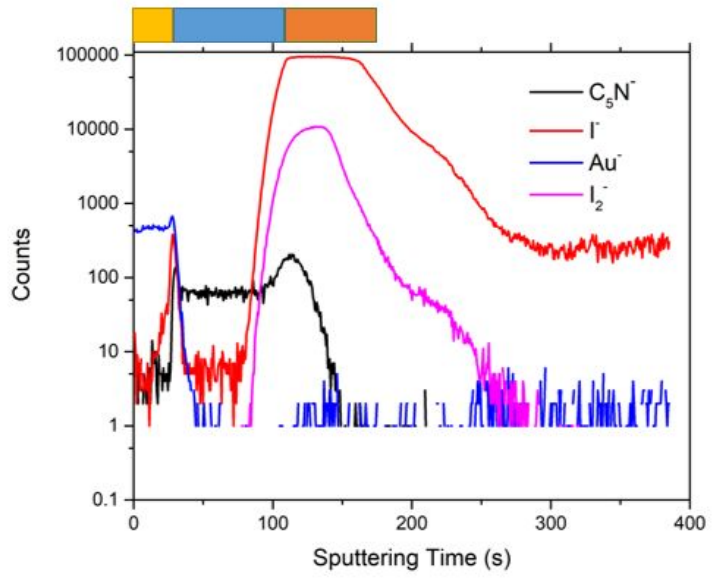

Figure S1. TOF-SIMS depth profile of 2.5D perovskite. a. fresh device. b. device after $40 \mathrm{~h}$ continuous operation at MPP point in $\mathrm{N}_{2}$ at room temperature. 
a

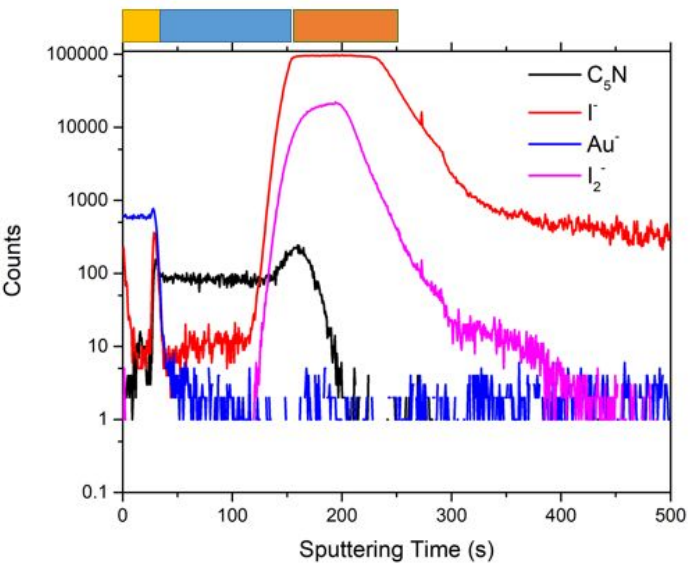

b

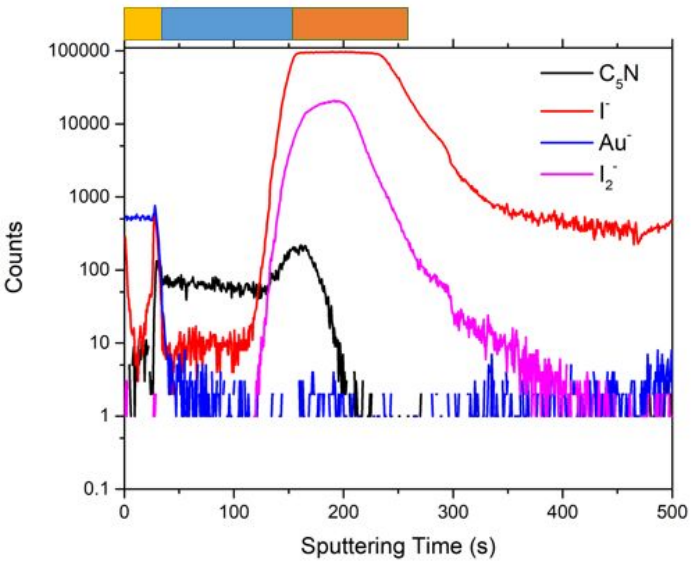

Figure S2. TOF-SIMS depth profile of 3D perovskite. a. fresh device. b. device after 40 h continuous operation at MPP point in $\mathrm{N}_{2}$ at room temperature. The only significant change is $\mathrm{I}^{-}$in the Au and spiro layers, especially at the $\mathrm{Au} /$ spiro interface. 
a

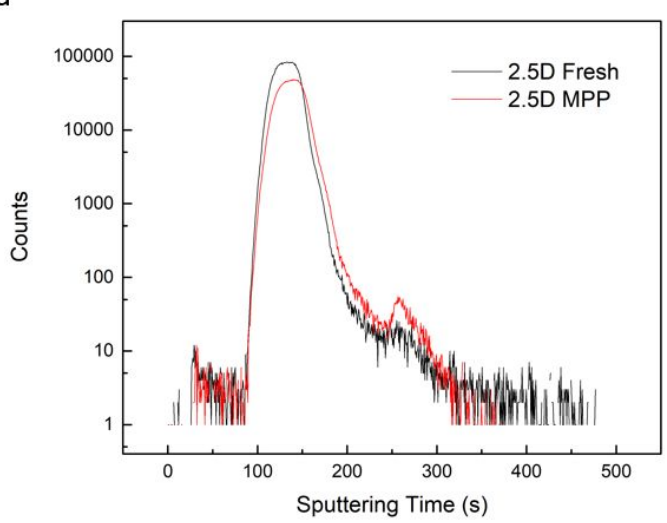

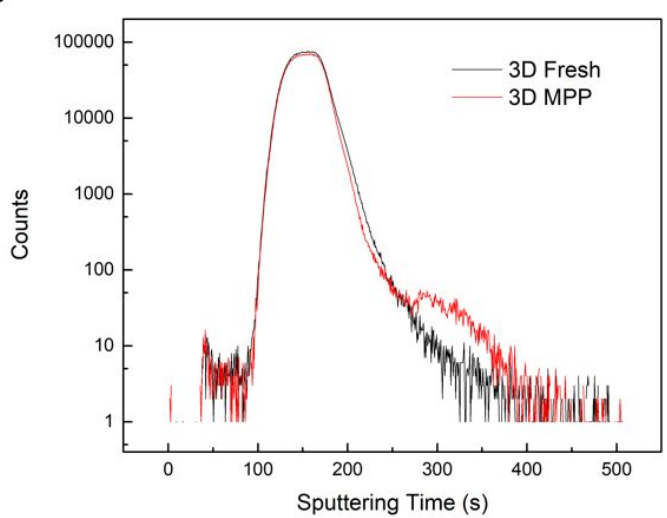

Figure S3. TOF-SIMS depth profile of Pb. a. fresh and MPP aged 2.5D devices, $b$. fresh and MPP aged 3D devices. 
a

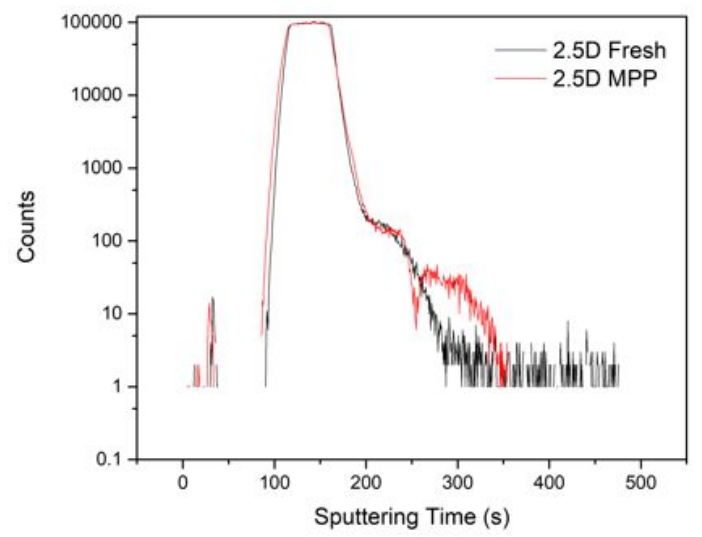

C

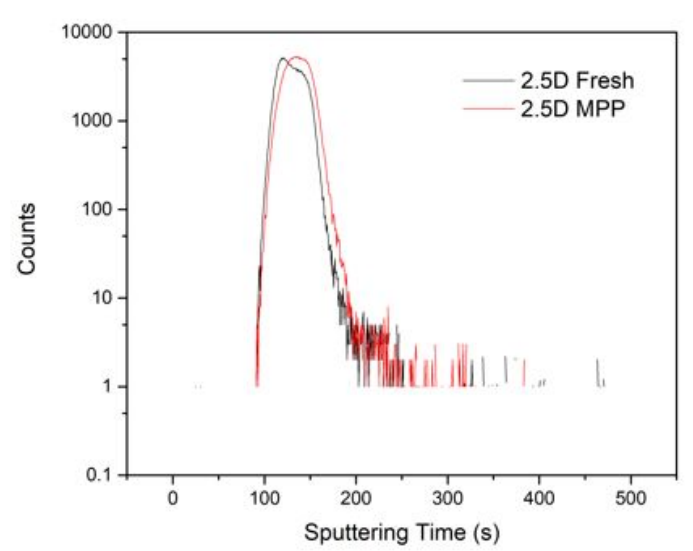

b

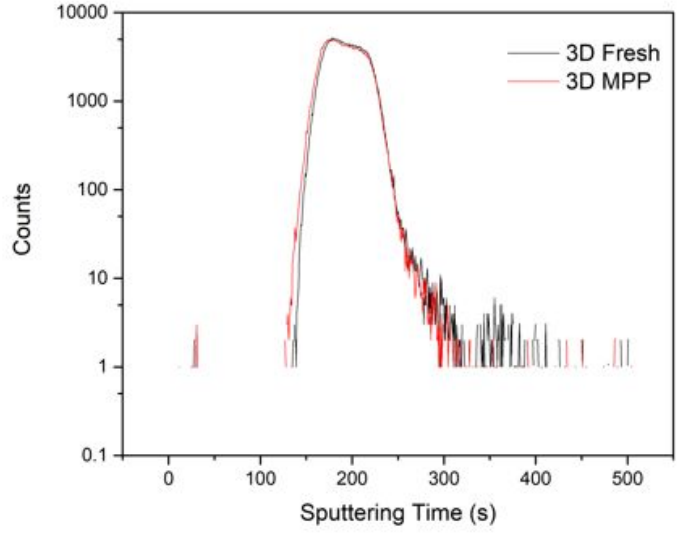

d

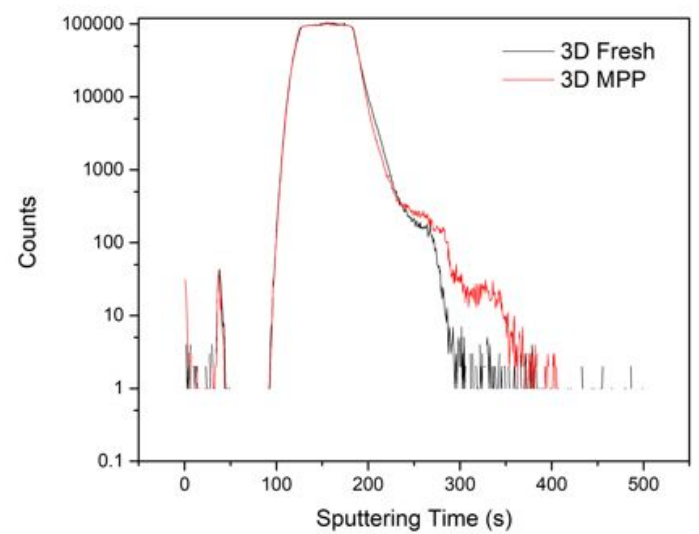

Figure S4. TOF-SIMS depth profile of FA $(a, b)$ and ${ }^{13}$ C-FA $(c, d)$. a, c. fresh and MPP aged 2.5D device, $\mathrm{b}, \mathrm{d}$. fresh and MPP aged 3D device. 
a

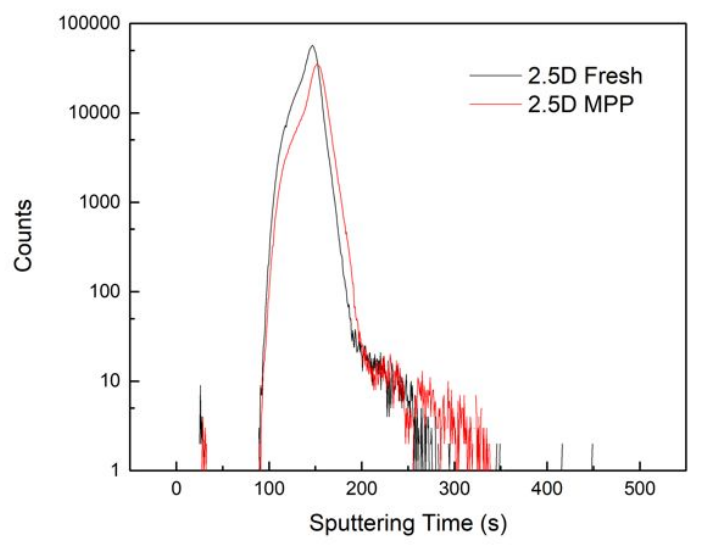

b

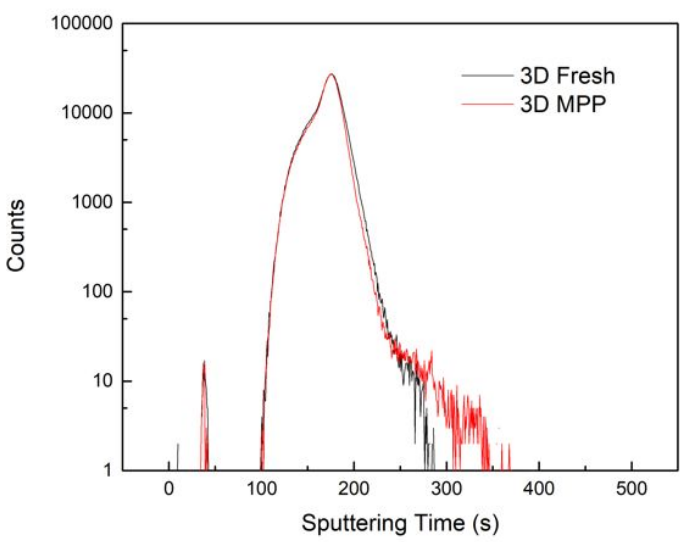

Figure S5. TOF-SIMS depth profile of MA. a. fresh and MPP aged 2.5D device, $b$. fresh and MPP aged $3 \mathrm{D}$ device. 
a

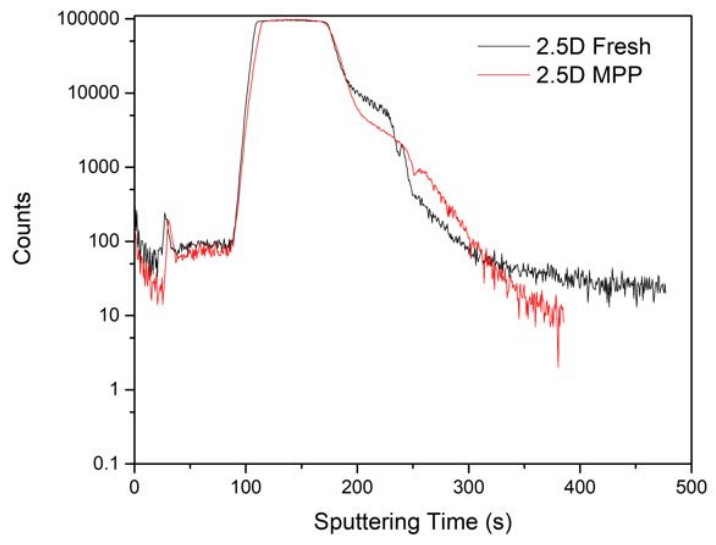

b

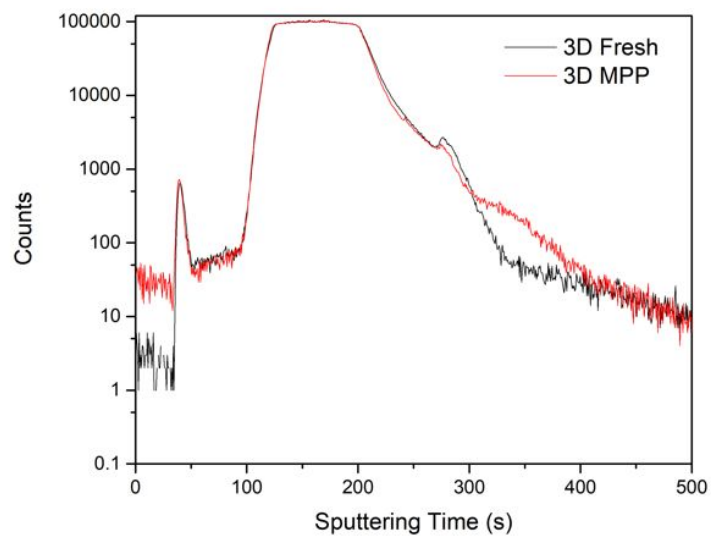

Figure S6. TOF-SIMS depth profile of Cs. a. fresh and MPP aged 2.5D device, b. fresh and MPP aged 3D device. 

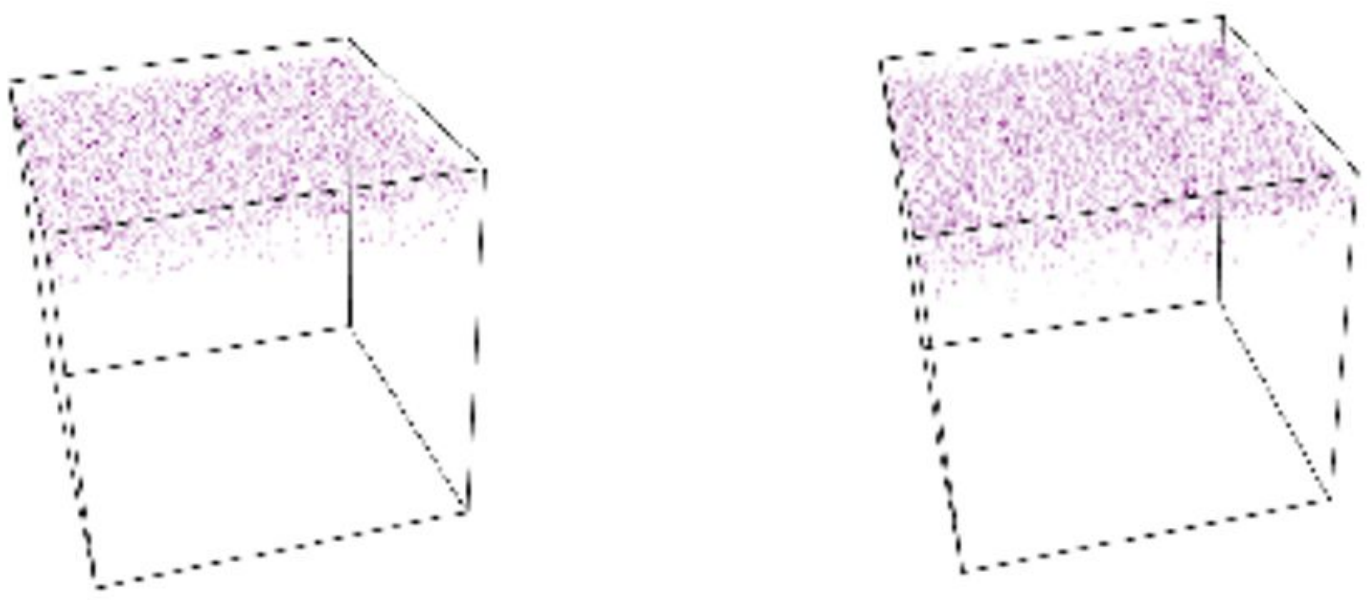

Figure S7. TOF-SIMS mapping of iodine signal in gold layer. a. Fresh 3D perovskite device. b. Fresh 2.5D perovskite device. 
a

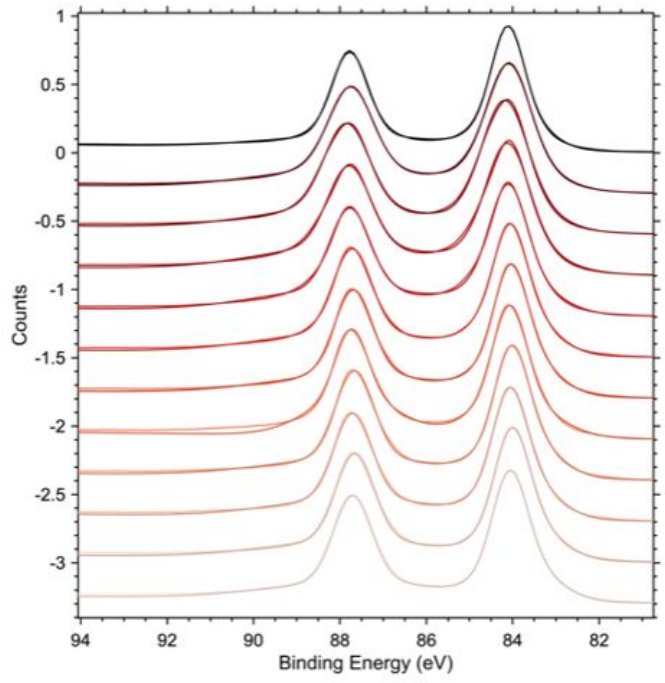

b

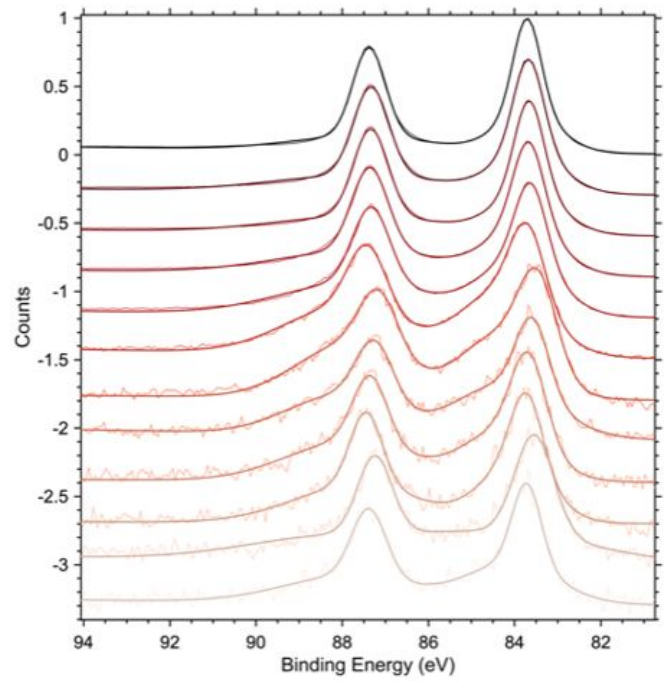

Figure S8. Sputtering x-ray photoelectron spectroscopy on a device pixel. a, fresh 3D device. b, aged 3D device. 


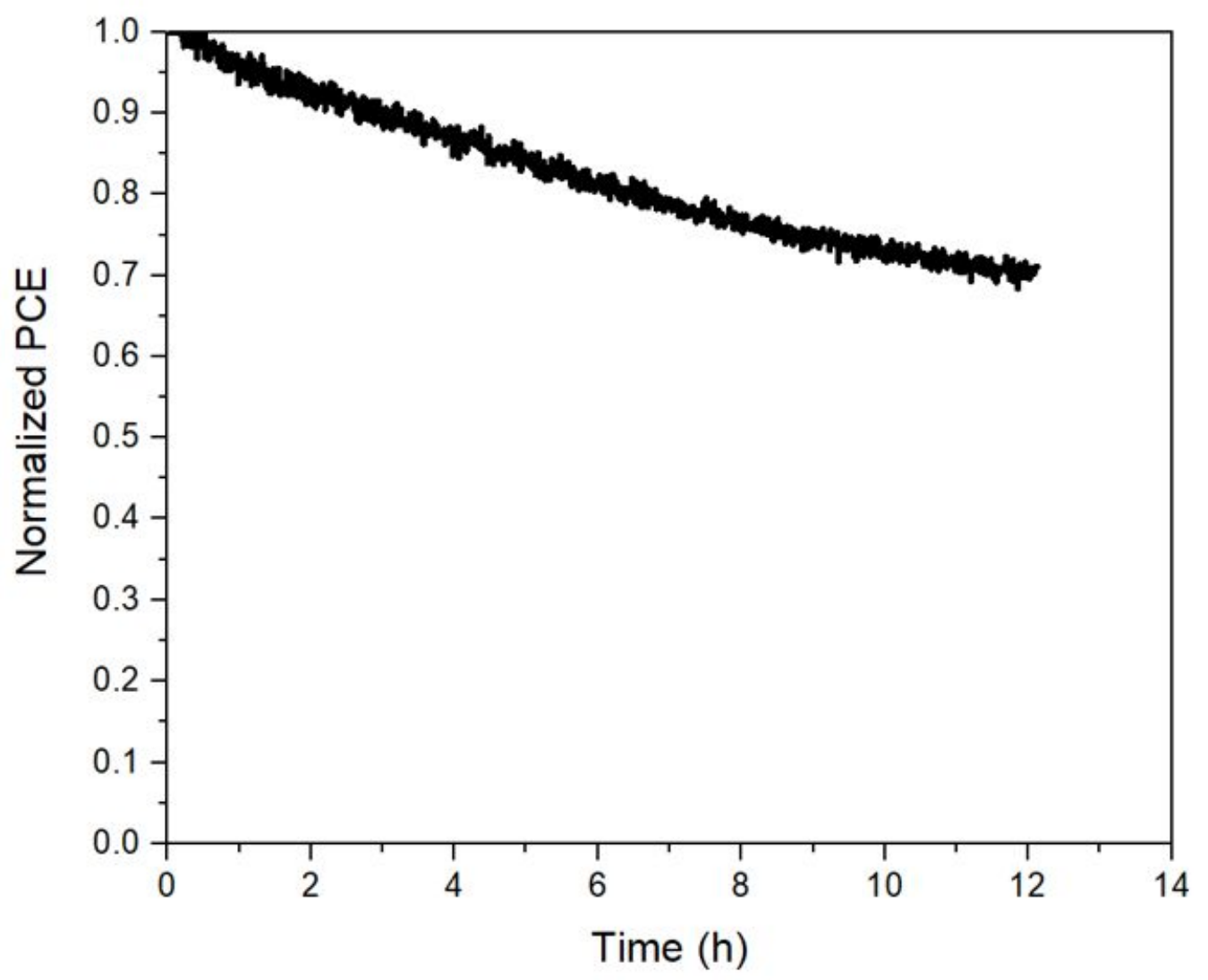

Fig. S9. Normalized PCE versus time for ALA post-treated 3D device. The device is aged under operating condition at MPP point.

\section{References}

(S1) Tan, H. et al. Efficient and stable solution-processed planar perovskite solar cells via contact passivation. Science 355, 722-726 (2017). 